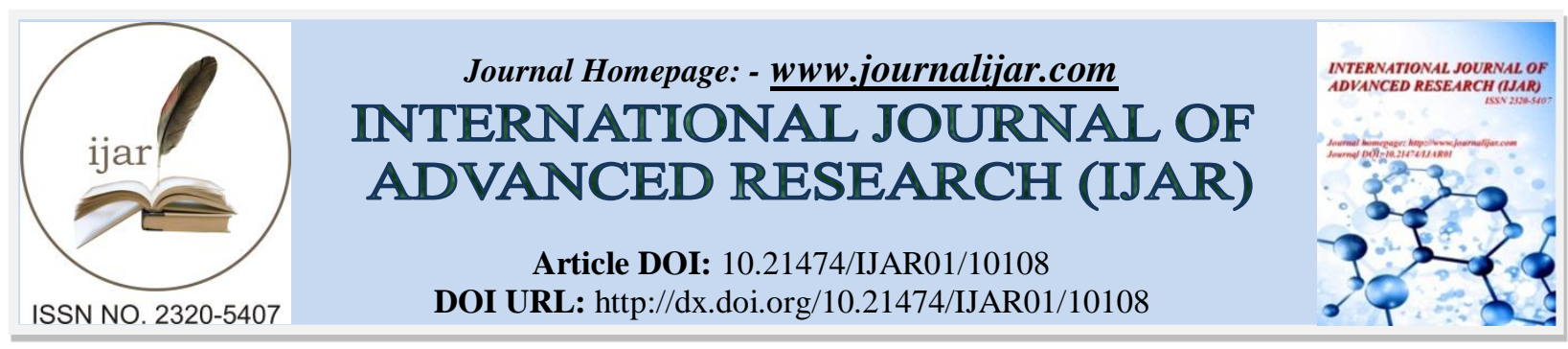

RESEARCH ARTICLE

\title{
SARCOÏDOSE MAMMAIRE : A PROPOS DE 2 CAS ET REVUS DE LA LITTERATURE.
}

\author{
L. Belachkar, A.Sounni, S Jayi , Fz Fdili, H Hikmat and Ma Melhouf. \\ Service de gynécologie-obstétrique II CHU HASSAN II FES.
}

\section{Manuscript Info}

\section{Manuscript History}

Received: 14 September 2019

Final Accepted: 16 October 2019

Published: November 2019

Key words:-

Sarcoïdose, sein, granulome épithéliocéllulaire.
Abstract

La sarcoïdose est une maladie inflammatoire granulomateuse, systémique, idiopathique, d'étiologie inconnue qui touche les deux sexes avec une prédominance chez la femme jeune. Elle est caractérisée histologiquement par la présence de granulome épithéliocellulaire sans nécrose caséeuse.

Elle atteint habituellement les poumons, la peau, le foie, la rate, les yeux, les phalanges des os et les glandes parotides.

La localisation mammaire est extrêmement rare et représente moins de $1 \%$ des cas,La mastite granulomateuse est une pseudotumeur inflammatoire bénigne. Cette entité anatomoclinique rare pose un problème de diagnostic différentiel avec d'autres granulomatoses et surtout avec le carcinome mammaire. La coexistence de manifestations systémiques doit faire discuter une sarcoïdose.

Dans notre travail nous rapportant 2 cas de sarcoïdose mammaire, qui ont été traité et guéri,

L'objectif de notre travail: Etablir les critères diagnostic de la mastite granulomateuse, et de citer les éléments majeur pour éliminer les autres diagnostic défferentiel surtout le carcinome mammaire ,

Copy Right, IJAR, 2019,. All rights reserved.

\section{Introduction:-}

La sarcoïdose est une maladie inflammatoire granulomateuse, systémique, idiopathique, d'étiologie inconnue qui touche les deux sexes avec une prédominance chez la femme jeune. Elle est caractérisée histologiquement par la présence de granulome épithéliocellulaire sans nécrose caséeuse.

Elle atteint habituellement les poumons, la peau, le foie, la rate, les yeux, les phalanges des os et les glandes parotides.

La localisation mammaire est extrêmement rare et représente moins de $1 \%$ des cas,La mastite granulomateuse est une pseudotumeur inflammatoire bénigne. Cette entité anatomo- clinique rare pose un problème de diagnostic différentiel avec d'autres granulomatoses et surtout avec le carcinome mammaire. La coexistence de manifestations systémiques doit faire discuter une sarcoïdose

\section{Observation:-}

Il s'agit d'une patiente de 37 ans ,G4P4, ayant un ATCD de mastite abcédé traité au cour de la première grossesse ,ayant consulté chez nous actuellement à 7 mois du post partum pour masse du sein gauche ;l'examen clinique ayant 
objectivé : sein bonnet B asymétriques ,SG : empâtement de la JQI de $3 / 4 \mathrm{~cm}$ avec fistulisation et issue du pus associé à une ADP axillaire homolatérale mobile de $15 \mathrm{~mm}, \mathrm{SD}$ sans anomalies .

Par ailleurs la patiente rapporte des signes systémique type arthralgies; chutes des cheveux.

L'échographie mammaire : plage hypoéchogène de la JQI du SG infiltrante présentant des contours irréguliers et comportant des logettes liquidienne classée ACR4 dont l'aspect échographique évoquant en premier une mastite suppurée.

Histologie: mastite granulomateuse épithéliode et gigantocelleure sans nécrose caséeuse.

La patiente a été mise sous corticoïde avec bonne évolution .

\section{Observation: num 2}

Mme S.N, âgée de 30ans, mariée et mère d'un enfant, ayant comme ATCD : mère suivi pour sarcoïdose pulmonaire, qui présente depuis 5 mois un nodule au niveau du sein gauche augmentant progressivement de volume associé à des arthralgies et des aphtes buccaux. L'examen trouve une tuméfaction au niveau du QSE faisant $2,5 / 2 \mathrm{~cm}$ indolore, dur, à contours irréguliers, avec rétraction cutanée, sans signes inflammatoires en regard, ni écoulement mamelonnaire. (Figure 1). Le sein controlatéral est sans particularités. Les aires ganglionnaires axillaires: libres. La mammographie + échographie mammaire :objective une lésion tissulaire, du QSE faisant $2 \mathrm{~cm}$, classée ACR4 avec adénopathie axillaire homolatérale de $1 \mathrm{~cm}$. (Figure2 ).

La patiente bénéficie d'une microbiopsie au trucut objectivant des cellules epithélio-giganto-cellulaires sans nécrose caséeuse en faveur d'une sarcoidose mammaire.

mise sous traitement corticoide systémique. L'évolution est marquée 3 mois après par la fistulisation à la peau. (Figure3).puis guérison complète.

\section{Figure 1:-}

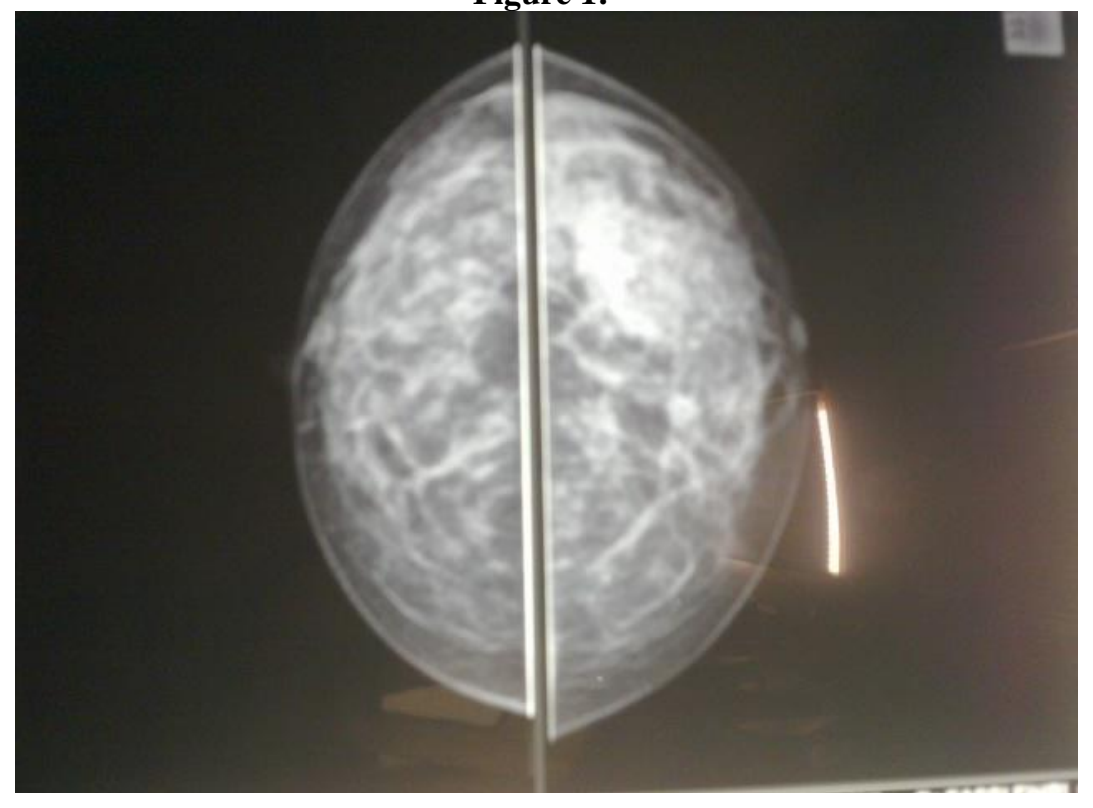




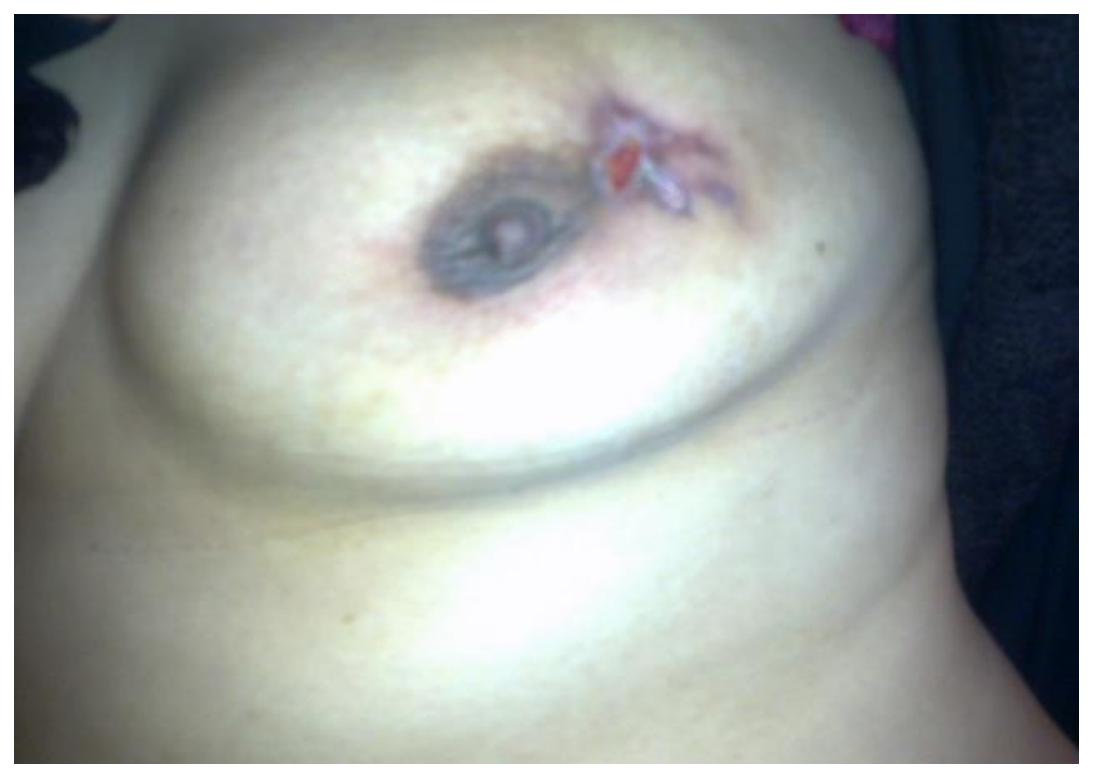

Figure 2:-

\section{Discussion:-}

Le diagnostic de la sarcoïdose repose sur un regroupement d'éléments cliniques, radiologiques, biologiques et histologiques dont aucun pris isolément n'est spécifique de la maladie et donc aucun n'est à la fois nécessaire et suffisant. En fonction de la définition actuellement admise le diagnostic se fond sur le regroupement de plusieurs données. 1. Arguments cliniques : Le jeune âge habituel du sujet atteint de la sarcoïdose, L'absence de notion de contage tuberculeux, La latence fonctionnelle habituelle du moins dans les formes récentes de la maladie, L'association de localisations intra-thoraciques et extra-thoraciques multiples et évocatrices telles : (cutanée spécifique, ophtalmologique, ganglionnaire ...) exprimant le caractère systémique de la maladie[1] .

Traitement Selon les cas, la sarcoïdose peut guérir sans traitement dans environ 50\% des cas, ou bien nécessiter un traitement qui peut être définitivement efficace en12 mois [2]. Dans certains cas, la corticothérapie doit être prolongée au-delà de 12 mois, voire indéfiniment.

Le traitement de référence demeure la prednisone.

la littérature a rapporté uniquement 35 cas de localisation mammaire de sarcoïdose histologiquement prouvés [6,4]. La localisation mammaire peut être primitive [5]. Cliniquement, elle se manifeste par des masses palpables au niveau des seins, dont la taille varie de $0,5 \mathrm{~cm}$ à $8 \mathrm{~cm}[5,6]$. Les résultats de l'imagerie en matière de sarcoïdose mammaire ont été rapportés dans un nombre limité de cas $[4,5,6]$ :

la mammographie a été rapportée dans neuf cas Dans trois cas, les contours de la masse étaient flous ou spiculés, simulant des lésions malignes ; deux mammographies étaient négatives, et, dans les autres cas, les résultats étaient non spécifiques ;la sarcoïdose mammaire peut donc souvent mimer le cancer mammaire, avec absence de microcalcifications le plus souvent[6];

l'échographie mammaire était non spécifique .

L' histologie confirme le diagnostic

\section{Conclusion:-}

La localisation mammaire de la sarcoïdose, malgré son incidence très rare, doit être évoquée dans le diagnostic différentiel du nodule mammaire, chez les femmes ayant des antécédents de sarcoïdose. L'IRM permet, grâce à sa valeur prédictive négative, d'écarter une lésion carcinomateuse. Cependant, l'histologie est habituellement exigée pour confirmer le diagnostic. 


\section{Bibliographie:-}

1. D Valeyre A Prasse H Nunes Sarcoidosis. Lancet 2014

2. MD Rossman E. Kreider Lesson learned from ACCESS (A Case Controlled Etiologic Study of Sarcoidosis). Proc Am Thor Soc 2007

3. Ishimaru K, Isomoto I, Okimoto T, Itoyanagi A, Uetani M. Sarcoidosis of the breast. Eur Radiol 2002

4. Ojeda H, Sardi A, Totoonchie A. Sarcoidosis of the breast: implications for the general surgeon. Am Surg 2013.

5. Takahashi R, Shibuya Y, Shijubo N, Asaishi K, Abe S. Mammary involvement in a patient with sarcoidosis. Intern Med 2000.

6. Gisvold JJ, Crotty TB, Johnson RE. Sarcoidosis presenting as spiculated breast masses. Mayo Clin Proc 2011. 